\title{
The RIB in-flight facility EXOTIC at INFN-LNL
}

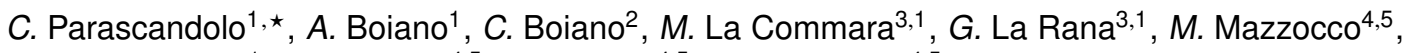 \\ D. Pierroutsakou ${ }^{1}$, C. Signorini ${ }^{4,5}$, F. Soramel $^{4,5}$, and E. Strano ${ }^{4,5}$ \\ ${ }^{1}$ INFN - Napoli, Via Cintia, I-80126 Napoli, Italy \\ ${ }^{2}$ INFN - Milano, Via Celoria 16, I-20133 Milano, Italy \\ ${ }^{3}$ Dipartimento di Fisica, Università di Napoli, Via Cintia, I-80126 Napoli, Italy \\ ${ }^{4}$ Dipartimento di Fisica e Astronomia, Università di Padova, Via Marzolo 8, I-35131 Padova, Italy \\ ${ }^{5}$ INFN - Padova, Via Marzolo 8, I-35131 Padova, Italy
}

\begin{abstract}
The EXOTIC facility, installed at the INFN-Laboratori Nazionali di Legnaro (LNL), is devoted to the in-flight production of light Radioactive Ion Beams (RIBs) in the energy range between 3-5 MeV/nucleon. The research field accessible using the EXOTIC RIBs is the investigation of reaction mechanisms and nuclear structure, resonant scattering measurements and measurements of nuclear reaction cross sections of astrophysical interest.
\end{abstract}

\section{Introduction}

During the last decades there has been a growing interest in the possibility of using Radioactive Ion Beams (RIBs) for a variety of fundamental studies in physics. Experiments with RIBs allow to explore the properties of isotopes with a proton-to-neutron ratio very different from the stable ones, measure reaction cross sections of astrophysical interest occurring in explosive environments, constrain the isospin-dependent nucleon-nucleon interaction in neutron-rich nuclei and in neutron stars, synthesize superheavy elements and test physics beyond the standard model.

\section{EXOTIC facility and Experimental Set-up}

The EXOTIC facility [1-4] is dedicated to the in-flight production of low-energy light RIBs, by inverse kinematics nuclear reactions using the intense heavy-ion beams from the INFN-LNL Tandem XTU accelerator hitting a light gas target $\left(\mathrm{H}_{2}, \mathrm{D}_{2},{ }^{3} \mathrm{He}\right.$ and $\left.{ }^{4} \mathrm{He}\right)$. The main features of the facility, shown in Fig. 1, are a large RIB acceptance of the optics elements and a large capability to suppress all the undesired scattered beams. It consists of: i) a production gas target that is a $5 \mathrm{~cm}$-long cylindrical cell walled with windows made of $2.2 \mu \mathrm{m}$ thick Havar foil, operating at room or at liquid $\mathrm{N}_{2}$ temperatures at pressures up to $1 \mathrm{~atm}$; ii) a beam selection and transport system consisting of: a triplet of large diameter quadrupole lenses, a $30^{\circ}$ bending magnet, a Wien filter and a second triplet of quadrupole lenses placed before the reaction chamber. The Wien filter eliminates to a very large extent the tails of the primary beam that pass through the system with the same magnetic rigidity.

^e-mail: concetta.parascandolo@na.infn.it 
In order to stop the RIB contaminants, different slit sets are installed along the beamline and can be adjusted to the envelope of the produced RIB. The facility has a length of $8.34 \mathrm{~m}$ and presents the following charateristics: $\Delta \mathrm{E} / \mathrm{E}= \pm 10 \%, \Delta \mathrm{p} / \mathrm{p}= \pm 5 \%, \Delta \theta= \pm 50 \mathrm{mrad}, \Delta \phi= \pm 65 \mathrm{mrad}, \Delta \Omega \approx 10 \mathrm{msr}$, $\mathrm{B} \rho=0.98 \mathrm{Tm}$. So far, RIBs of ${ }^{7} \mathrm{Be},{ }^{8} \mathrm{~B},{ }^{17} \mathrm{~F},{ }^{15} \mathrm{O},{ }^{8} \mathrm{Li},{ }^{10} \mathrm{C}$ and ${ }^{11} \mathrm{C}$ at $3-5 \mathrm{MeV} /$ nucleon have been delivered with intensities about $10^{6}, 10^{3}, 10^{5}, 4 \times 10^{4}, 10^{5}, 5 \times 10^{3}$ and $2 \times 10^{5} \mathrm{pps}$, respectively, and with 98-99\% purity (except for ${ }^{8} \mathrm{~B}$ that has a lower purity).

$1-1^{\text {st }}$ slit system

2 - production gas target

$3-1^{\text {st }}$ quadrupole triplet

$4-2^{\text {nd }}$ slit system

$5-30^{\circ}$ analysing magnet

$6-3^{\text {rd }}$ slit system

7 - Wien filter

$8-2^{\text {nd }}$ quadrupole triplet

9 - 4th slit system

10 - reaction chamber

\section{primary beam}

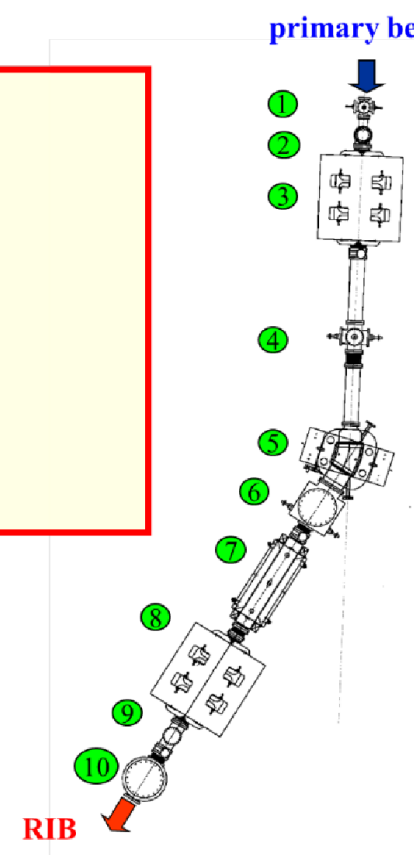

Figure 1. Layout of the EXOTIC facility.

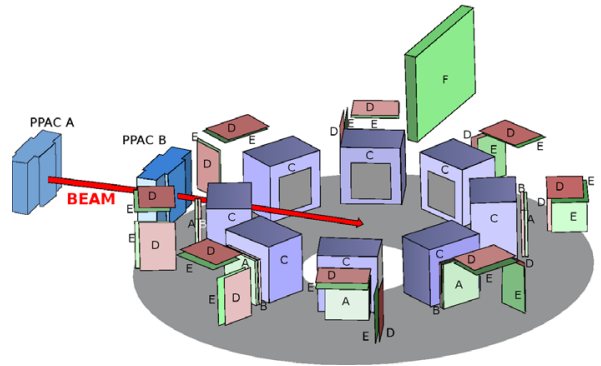

Figure 2. The position sensitive PPACs for the RIB tracking system and the detection system, EXPADES, placed in the reaction chamber (element 10 of Fig. 1) at the end of the EXOTIC facility.

The experimental set-up [5] of the EXOTIC facility, shown in Fig. 2 and situated at the end of the beamline, consists of: a) the RIB tracking system and b) the detection system, EXPADES, a new charged-particle telescope array. EXPADES is flexible to suit many experiments and it can be coupled with $\gamma$-ray and neutron arrays.

The Parallel Plate Avalanche Counters (PPACs) of the tracking system are position-sensitive and fast detectors, which can sustain counting rates up to $\sim 10^{6} \mathrm{~Hz}$. They are placed $909 \mathrm{~mm}$ (PPAC A) and $365 \mathrm{~mm}$ (PPAC B) upstream the reaction target, at the EXOTIC focal plane. The PPACs are filled with $\mathrm{C}_{4} \mathrm{H}_{10}$ gas at a working pressure of 10-20 mbar. Each PPAC has an active area of $62 \times 62 \mathrm{~mm}^{2}$ and is sealed with $1.5 \mu \mathrm{m}$-thick mylar windows. The detector has a central cathode and two anodes, placed symmetrically at $2.4 \mathrm{~mm}$ from the cathode. The position information of a particle crossing the PPAC is extracted from the anode signals by using a delay-line readout. The $1 \mathrm{~mm}$ resolution of the two PPACs allows us to reconstruct the position of the event on the reaction target with a $2.3 \mathrm{~mm}$ position resolution. The cathode signal is used as a reference time for Time of Flight measurements (giving a START signal with $0.9 \mathrm{~ns}$ FWHM time resolution) and for trigger purposes.

EXPADES is an array of eight telescopes arranged in a cylindrical configuration around the reaction target (Fig. 2). The telescope structure is flexible and is composed of two Double Side Silicon 
Strip Detectors (DSSSDs) and/or Ionization Chamber (IC), depending on the experimental requests. We use 40/60 $\mu \mathrm{m}$-thick DSSSDs for the $\Delta \mathrm{E}$ stage (B in Fig.2) and $300 \mu \mathrm{m}$-thick DSSSDs for the $\mathrm{E}_{\text {res }}$ one (A in Fig.2). Each DSSSD has 64-mm long 32x32 strips, with $2 \mathrm{~mm}$ pitch size and $40 \mu \mathrm{m}$ interstrip separation, defining thus a $\sim 2 \times 2 \mathrm{~mm}^{2}$ pixel structure. For experiments requiring the detection of more energetic particles than those stopped in the $\mathrm{E}_{r e s}, 1 \mathrm{~mm}$-thick DSSSDs can substitute the 300 $\mu \mathrm{m}$-thick ones or can be used in addition to the previous stages. ASIC-based electronics was employed for the treatment of the $\mathrm{E}_{\text {res }}$ signals, with a high granularity and a very low cost. For the signal readout of the $\Delta \mathrm{E}$ stage a compact low-noise electronics with very good energy and timing characteristics was developed by our collaboration. A valid alternative to allow the $\Delta \mathrm{E}-\mathrm{E}_{\text {res }}$ identification of reaction products with range in silicon shorter than $40 / 60 \mu \mathrm{m}$ is the use of a transverse field IC (C in Fig. 2) that can give the possibility to tune the effective thickness of the $\Delta \mathrm{E}$ layer by changing the gas pressure. The IC has $65 \times 65 \mathrm{~mm}^{2}$ entrance and exit windows $(1.5 \mu \mathrm{m}$-thick mylar) and can be filled with $\mathrm{CF}_{4}$ gas at pressures up to 100 mbar. The low-noise charge-sensitive preamplifiers for the $\Delta \mathrm{E}$ DSSSDs (D in Fig. 2), those of the ICs (not present in Fig. 2) as well as the electronic boards (E in Fig. 2) for the $\mathrm{E}_{\text {res }}$ DSSSDs are placed under vacuum close to the detectors. In this way, we manage to keep as low as possible the DSSSDs electronic thresholds, typically $300-500 \mathrm{keV}$.

\section{Experimental program and perspectives}

The experimental program at EXOTIC is varied and aims at different topics.

Firstly, we focused our attention on the study of reaction mechanisms induced by light radioactive (exotic) nuclei impinging on medium- and heavy-mass targets at energies near to the Coulomb barrier [6-10]. Indeed, the peculiar features of exotic nuclei influence the reaction mechanisms giving a picture that is rather different from that of well bound species [11]. In these measurements the charged products emitted in direct nuclear reactions and the light charged particles emitted in fusion-evaporation reactions should be charge and mass identified.

Furthemore, we started to study $\alpha$ clustering phenomena in light exotic nuclei [12], employing the Thick Target Inverse Kinematics (TTIK) scattering technique [13], with the RIB impinging on a ${ }^{4} \mathrm{He}$ gas target. The pressure of the gas is tuned such that the RIB completely stops in the gas while the energetic recoiling light target nuclei, due to their low rate of energy loss, can traverse the gas and be detected. The TTIK method is useful for measurements with low-intensity RIBs since it allows to measure the elastic scattering excitation function and the reduced $\alpha$-width directly, over a wide energy range by using a single beam energy, since the gas acts as a target but also as a degrader. In particular, we searched for $\alpha$-cluster states in ${ }^{19} \mathrm{Ne}$ above its $\alpha$-decay threshold measuring, for the first time, the ${ }^{15} \mathrm{O}\left({ }^{4} \mathrm{He},{ }^{4} \mathrm{He}\right){ }^{15} \mathrm{O}$ elastic scattering excitation function with the TTIK technique with ${ }^{15} \mathrm{O}$ RIB produced at EXOTIC. ${ }^{19} \mathrm{Ne}$ has attracted much interest due to the astrophysical importance of the ${ }^{15} \mathrm{O}(\alpha, \gamma){ }^{19} \mathrm{Ne}$ reaction, identified as a break-out from the Hot CNO cycle and a trigger for the following $r p$-capture populating masses higher than neon [14]. Neon isotopes exhibit strong $\alpha$ clustering phenomena, even in the ground states. Therefore, the idea has been to investigate the possible cluster nature of the neutron-poor side of the neon isotopes, almost totally unknown. The final excitation functions reveal the presence of resonances, that have been analysed by using a full R-Matrix fit with Azure2 [15].

Another possibility offered by the EXOTIC facility is to perform measurements of astrophysical interest with RIBs impinging on solid or gas light targets in inverse kinematics. Among the different processes of stellar nucleosynthesis forming elements heavier than ${ }^{9} \mathrm{Be}$, the $r p$-capture and $\alpha \mathrm{p}$ processes, occurring in explosive astrophysical environments, are those than can be investigated by using the EXOTIC RIBs. By developing a radioactive ${ }^{18} \mathrm{Ne}$ beam through the ${ }^{3} \mathrm{He}\left({ }^{16} \mathrm{O},{ }^{18} \mathrm{Ne}\right) \mathrm{n}$ reaction, the ${ }^{18} \mathrm{Ne}(\alpha, \mathrm{p})^{21} \mathrm{Na}$ process could be studied at astrophysical energies to provide a link between the Hot 
$\mathrm{CNO}$ cycle and the $r p$-process [14]. Another measurement relevant to astrophysics can be performed at EXOTIC such as the ${ }^{30} \mathrm{P}(\mathrm{p}, \gamma){ }^{31} \mathrm{~S}$ with a ${ }^{30} \mathrm{P}$ beam, essential for the production of heavy elements (from $\mathrm{Si}$ to $\mathrm{Ca}$ ) in the explosion of $\mathrm{ONe}$ novae and in particular to explain the anomalously high ${ }^{30} \mathrm{Si} /{ }^{28} \mathrm{Si}$ rate measured in pre-solar grains of possible ONe novae origin [16]. Moreover, experiments based on the Trojan Horse Method (THM) [17] are considered. In this direction, a first experiment was performed by means of the THM for the ${ }^{7} \mathrm{Be}(\mathrm{n}, \alpha)^{4} \mathrm{He}$ reaction to shed light on the cosmological lithium-problem, of interest in the Big Bang Nucleosynthesis [18]. The first results have been presented in this conference [19].

Recently, we tested also the possibility to perform measurements of fusion cross sections at nearand sub-barrier energies, by using our facility as a separator of evaporation residues from the incident beam, provided by the INFN-LNL Tandem XTU accelerator. The evaporation residues are transported and detected at the focal plane of the facility (for details, see [20]).

To summarize, the EXOTIC facility dedicated to the in-flight production of low-energy light RIBs and its experimental set-up is fully operational at INFN-LNL. Stimulating nuclear physics and nuclear astrophysics measurements can be carried out employing the produced RIBs, in the framework of international collaborations. Moreover, EXOTIC can be used as a velocity filter to perform fusionevaporation experiments at near-and sub-barrier energies with stable beams and also with the neutronrich RIBs delivered by the next generation ISOL-type facility SPES [21], in construction at INFNLNL.

\section{References}

[1] V.Z. Maidikov et al., Nucl. Phys. A 746, 389c (2004);

[2] D. Pierroutsakou et al., Eur. Phys. J. Special Topics 150, 47 (2007);

[3] F. Farinon et al., Nucl. Instr. and Meth. B 266, 4097 (2008);

[4] M. Mazzocco et al., Nucl. Instr. and Meth. B 266, 4665 (2008) and Nucl. Instr. and Meth. B 317, 223 (2013)

[5] D. Pierroutsakou et al., Nucl. Instr. and Meth. A 834, 46 (2016)

[6] C. Signorini et al., Eur. Phys. J. A 44, 63 (2010)

[7] M. Mazzocco et al., Phys. Rev. C 82, 054604 (2010), Phys. Rev. C 92, 024615 (2015); EPJ Web of Conferences 117, 06006 (2016)

[8] N. Patronis et al., Phys. Rev. C 85, 024609 (2012); A. Pakou et al., Phys. Rev. C 87, 014619 (2013); Eur. Phys. J. A 51, 55 (2015); Eur. Phys. J. A 51, 90 (2015)

[9] E. Strano et al., Phys. Rev. C 94, 024622 (2016)

[10] O. Sgouros et al., Phys. Rev. C 94, 044623 (2016); Phys. Rev. C 95, 054609 (2017)

[11] L.F. Canto et al., Phys. Rep. 424, 1 (2006); Phys. Rep. 596, 1 (2015)

[12] M. Freer, Rep. Prog. Phys. 70, 2149221 (2007)

[13] K. Artemov et al., Sov. J. Nucl. Phys. 52, 408-411 (1990)

[14] M. Wiescher et al., Prog. Part. and Nucl. Phys. 59, 51 (2007)

[15] D. Torresi et al., J. Phys.: Conf. Ser. 863, 012026 (2017); Phys. Rev. C 96, 044317 (2017)

[16] J. José et al., Astrophys. J. 560, 897 (2001)

[17] G. Baur, Phys. Lett. B 178, 135 (1986); R. E. Tribble et al., Rep. Prog. Phys. 77, 106901 (2014)

[18] C. Broggini et al., J. Cosmol. Astropart. Phys. 6, 30 (2012)

[19] L. Lamia et al., to be published

[20] E. Strano et al., Nucl. Instr. and Meth. A 877, 293 (2018)

[21] https://web.infn.it/spes/ 